Supplement of

\title{
A global satellite environmental data record derived from AMSR-E and AMSR2 microwave earth observations
}

5 Jinyang Du et al.

Correspondence to: Jinyang Du (jinyang.du@ntsg.umt.edu)

10

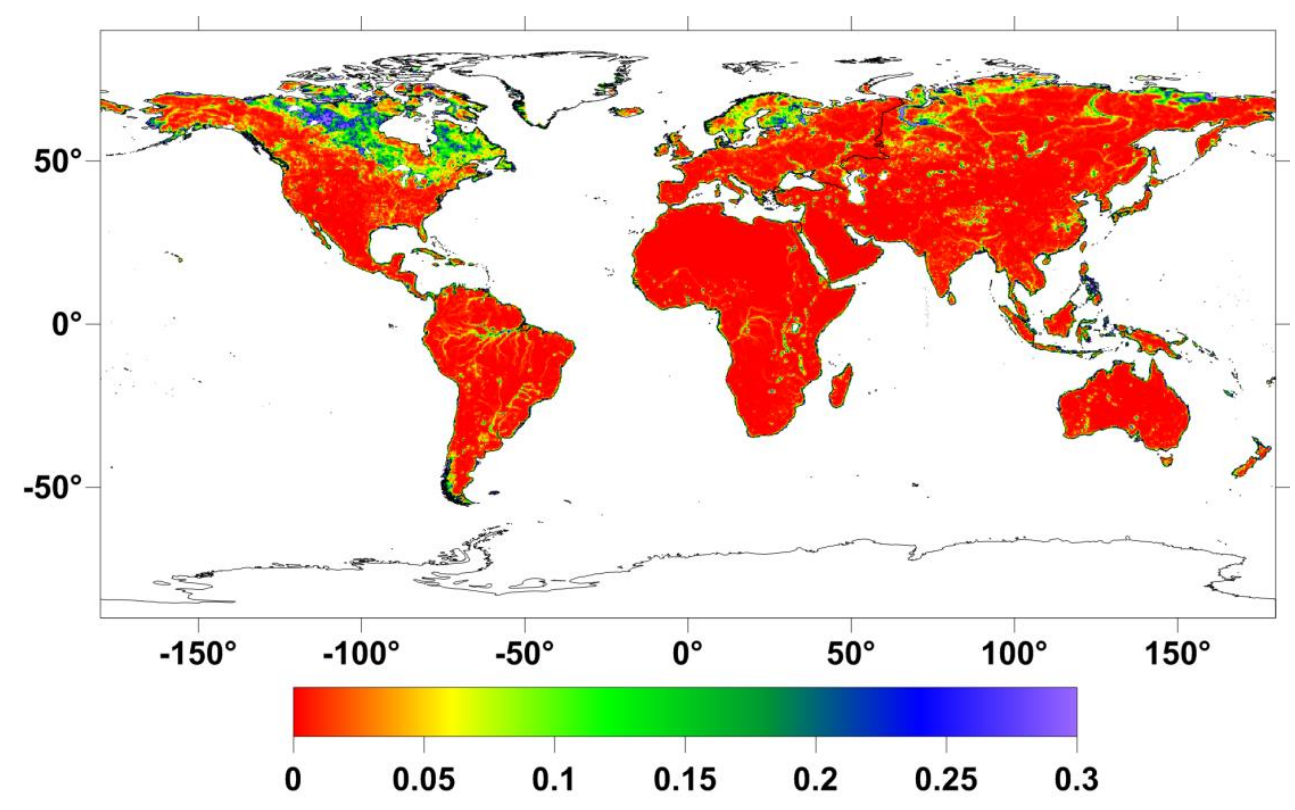

Supplementary Figure 1: The 25-km MOD44W static water map aggregated from original 250-m resolution. 

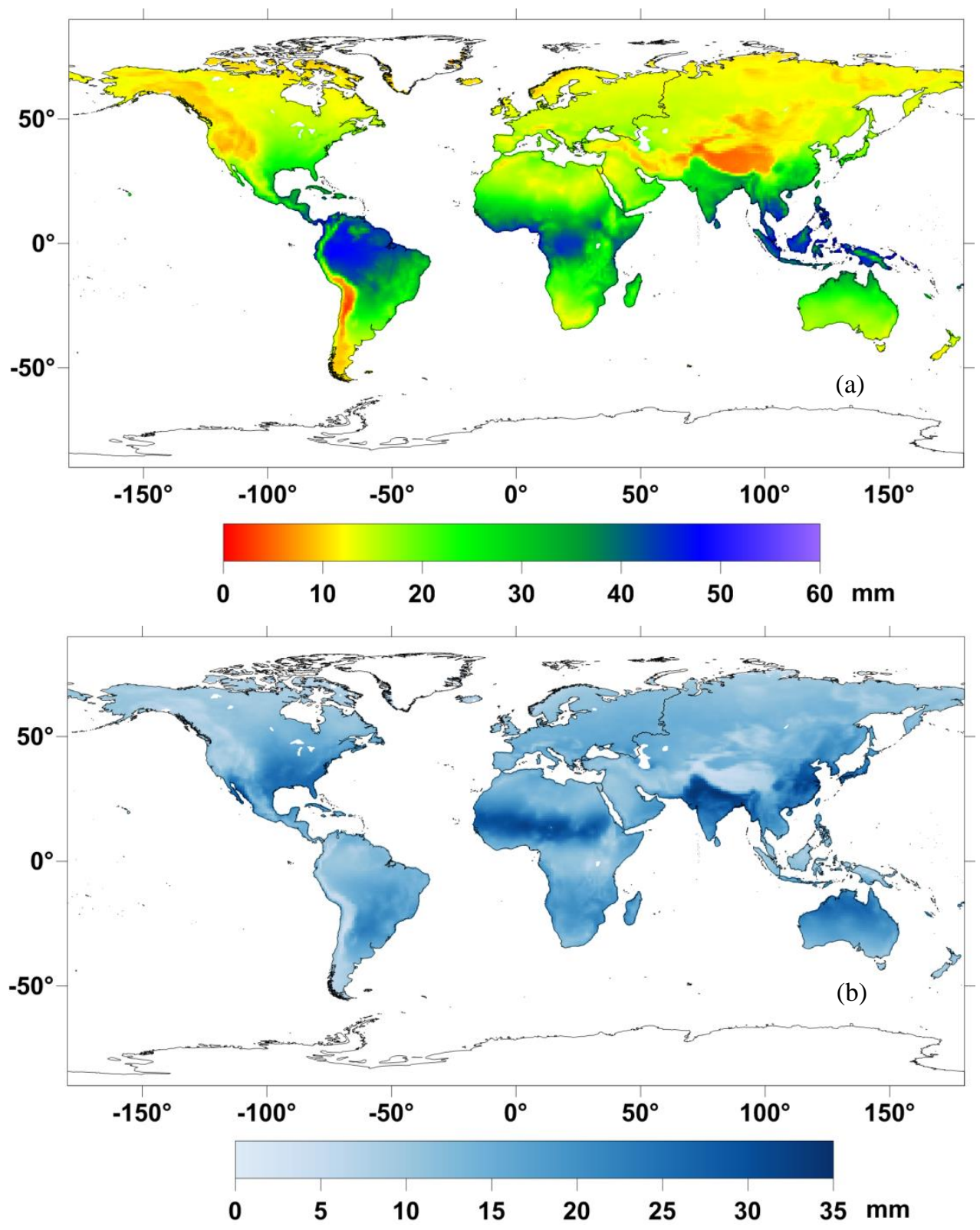

Supplementary Figure 2: AIRS climatology atmosphere precipitable water vapor $(P W V)$ mean (a) and variability (standard deviation) (b) for years 2003-2010 and 2013-2015. 

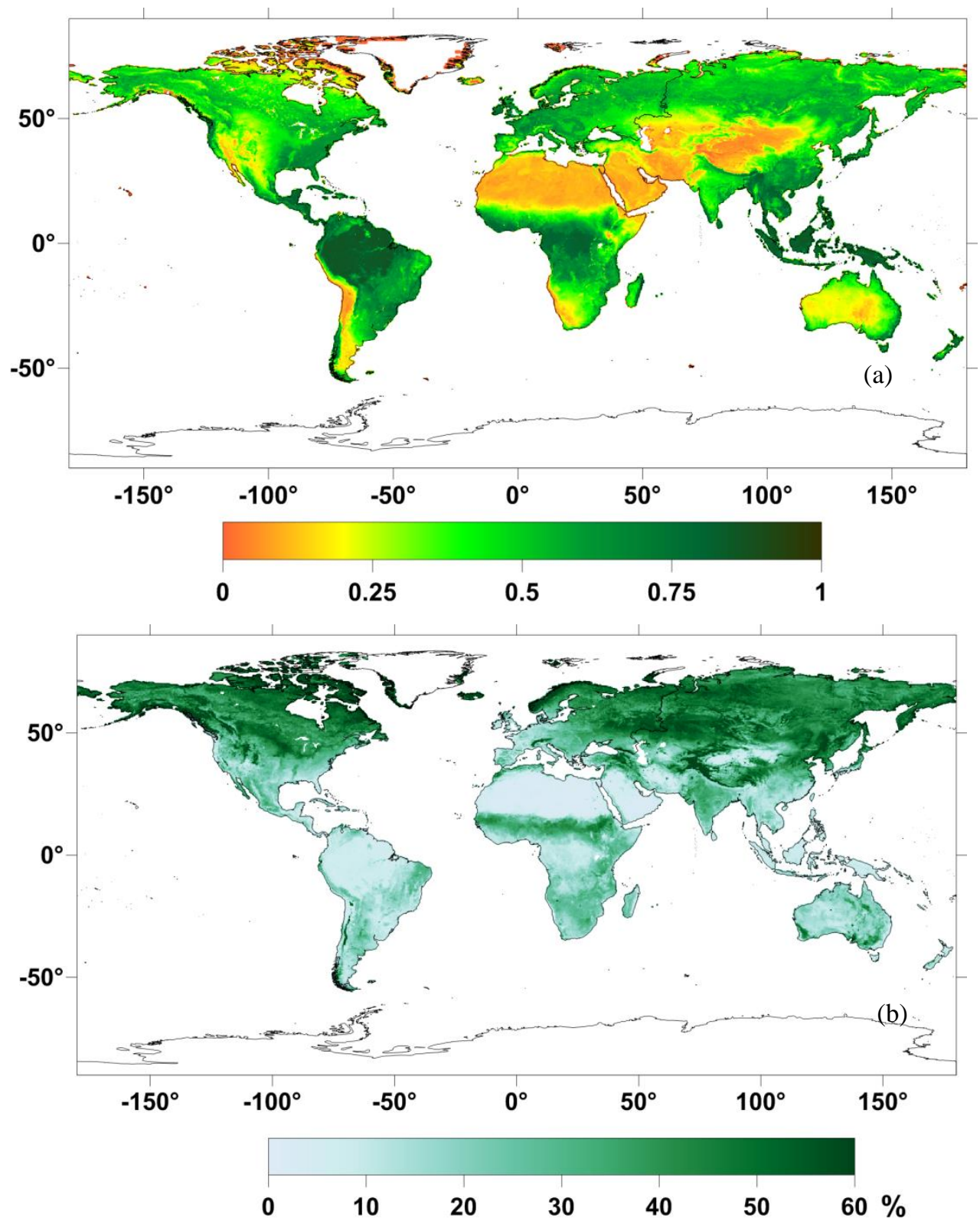

5 Supplementary Figure 3: Annual mean (a) and seasonal SD variability (b) of GIMMS3g NDVI over years 2003-2010 and 20132015 
Supplementary Table 1: Statistical mean, temporal standard deviation (SD) and temporal range calculated from LPDR monthly

5 composites over respective full-year AMSR-E (2003-2010) and AMSR2 (2013-2015) portions of record. The LPDR parameters analyzed are separated by ascending and descending orbit retrievals for fractional water $(f w)$, precipitable water vapor $(P W V)$, daily maximum/minimum surface air temperature (Tmx and Tmn), X-band vegetation optical depth (VOD) and surface volumetric soil moisture (vsm).

\begin{tabular}{|c|c|c|c|c|c|c|}
\hline \multirow[b]{2}{*}{ Ascending } & \multicolumn{2}{|c|}{ Mean } & \multicolumn{2}{|c|}{ SD } & \multicolumn{2}{|c|}{ Range } \\
\hline & AMSR-E & AMSR2 & AMSR-E & AMSR2 & AMSR-E & AMSR2 \\
\hline$f w$ & 0.04 & 0.04 & 0.00 & 0.00 & 0.01 & 0.01 \\
\hline$P W V(\mathrm{~mm})$ & 20.87 & 20.37 & 1.79 & 1.45 & 5.46 & 4.66 \\
\hline $\operatorname{Tm} x\left({ }^{\circ} \mathrm{C}\right)$ & 25.81 & 25.57 & 1.39 & 1.32 & 4.68 & 4.31 \\
\hline$V O D$ & 1.03 & 1.00 & 0.06 & 0.06 & 0.17 & 0.15 \\
\hline $\operatorname{vsm}\left(\mathrm{cm}^{3} / \mathrm{cm}^{3}\right)$ & 0.14 & 0.13 & 0.01 & 0.01 & 0.03 & 0.03 \\
\hline Descending & AMSR-E & AMSR2 & AMSR-E & AMSR2 & AMSR-E & AMSR2 \\
\hline$f w$ & 0.04 & 0.04 & 0.01 & 0.01 & 0.02 & 0.02 \\
\hline$P W V(\mathrm{~mm})$ & 22.30 & 21.85 & 1.67 & 1.41 & 5.28 & 4.85 \\
\hline $\operatorname{Tmn}\left({ }^{\circ} \mathrm{C}\right)$ & 13.53 & 13.66 & 1.14 & 1.12 & 3.84 & 3.87 \\
\hline$V O D$ & 1.01 & 1.02 & 0.08 & 0.07 & 0.20 & 0.17 \\
\hline$v s m\left(\mathrm{~cm}^{3} / \mathrm{cm}^{3}\right)$ & 0.14 & 0.15 & 0.01 & 0.01 & 0.03 & 0.03 \\
\hline
\end{tabular}

